\title{
IMPULSIVE INTERMODAL CYBER BULLYING RECOGNITION FROM PUBLIC NETS
}

\author{
Pradheep.T \\ PG Student, \\ Department of Computer Science and Engineering \\ Pondicherry Engineering College \\ Puducherry,India \\ Yogeshwaran.T \\ UG Student, \\ Department of Information Technology \\ Pondicherry Engineering College \\ Puducherry,India
}

\author{
J.I.Sheeba \\ Assistant Professor, \\ Department of Computer Science and Engineering \\ Pondicherry Engineering College \\ Puducherry,India \\ S. Pradeep Devaneyan \\ Dean, School of Mechanical and \\ Building Sciences Christ College of Engineering \\ and Technology \\ Puducherry,India
}

\begin{abstract}
Cyberbullying has grown as an important societal challenge nowadays. The Cyberbullying affects both in terms of psychological and emotional means of a person. So there is a need to devise a method to detect and prevent cyberbullying in social networks. Most of the existing cyberbullying methods involves only text detection and few methods are available for analysing the visual detection. In this proposed work is going to detect multimodel cyberbullying such as audio, video, image along with text in the social networks. The cyberbully image will be detected using the computer vision algorithm which includes two methods like Image Similarity and Optical Character Recognition (OCR). The cyberbully video will be detected using the Shot Boundary detection algorithm where the video will be broken into frames and analysed using various methods in it. The proposed framework also support to identify the cyberbully audio in the social network. Finally the cyberbully data will be classified into Physical bullying, Social bullying and Verbal bullying using classifiers.
\end{abstract}

Keywords: Cyberbully Detection, Social Networks, Physical bullying, Social bullying, Verbal bullying, Classifiers.

\section{I.INTRODUCTION}

The Social networking platform has become very popular in the last few years. Through this social media the people interact, share, discuss and disseminate knowledge for the benefit of others by using multimodal features like multimedia text, pictures, videos and audio. The users can create their own profiles and communicate with other users regardless of their presence in terms of location. Cyber criminals have used the social media as a new platform for committing different cybercrimes such [8] as phishing, spamming, spread of rumours, and cyberbullying.

Social media platforms have a huge global reach and its audience cover increases year after year, with YouTube boasting more than 1 billion users per month [9]. Similarly, Twitter has on average 500 million tweets per day, while Facebook remains the largest social media network with million active users and many million people sending updates. As this shows, cybercrime criminals have been using both platforms which have attracted thousands of views, comments, forums and posts. For example, through the use of videos, images posted on YouTube, Facebook and Instagram they try bully people. The videos and images attracts accompanied with taglines makes bullying easier to cause damage to people. Bullying is generally defined as repeated hostile behaviour. Bullying has included mainly physical acts, verbal abuse and social [3] exclusion. The increase of electronic communications technologies causes youth and children to undergo a new way of bullying. There are many various types of cyberbullying such as:Flaming,Harassment, Masquerading, Outing andExclusion. Reducing cyber harassment [3] is important because various negative health outcomes have been observed among people those who were affected by cyberbullying, such as depression, anxiety, loneliness, suicidal behaviour.

The main objective of this proposed work is to detect the cyberbully content in different forms such as image, audio, video and text in the social media. And classifying the content using naïve Bayesian classifier into three different kinds physical, social and verbal bullying. Thus preventing the users in social media to post any vulnerable content which affects the people in different ways. In this paper section 2 describes the related works, section 3 describes the proposed work and section 4 describes the conclusion of the paper.

\section{II.RELATED WORKS}

This section discuss the existing works available in cyberbullying detection from social networks. 
A.Habiba and J.I. Sheeba proposed studied about the detection of the general cyber bully polarity and devised methods like pre-processing, frequency measure and Classifier algorithm for extracting cyberbully polarity from blog messages [1]. J. I. Sheeba and K. Vivekanandan proposed a model for extracting cyber bully polarity from the Forum uses the methods of preprocessing, frequency calculation, noun extraction and Fuzzy C-means algorithm. Finally the results obtained are reduced by using Fuzzy Decision Tree methods and Fuzzy rules and they are evaluated by the existing systems like Naïve Bayes classifier and Mamdani fuzzy inference system [2]. J. I. Sheeba and B. Sri Nandhini proposed another model for detecting the presence of cyber bullying in social media using the Levenshtein algorithm [3]. J. I. Sheeba and B. Sri Nandhini proposed a model for detecting the presence of cyber bullying activity in social networks using fuzzy logic. The system also uses genetic operators like crossover and mutation for optimizing the parameters and obtains precise type of cyber bullying activity [4]. Krishna B.Kansara and Narendra M. Shekokar have proposed a framework for the purpose of detecting negative online interactions in terms of abusive contents carried out through text messages as well as images [5]. Mohammed Ali, et al., have proposed a model of supervised machine learning solution for detecting cyberbullyingin the Twitter. Where a set of unique features derived from Twitter; network, activity, user, and tweet content, based on which the feasible solution to detecting Cyberbullying was provided [6].J.I. Sheeba and S. Pradeep Devaneyanproposed a model to detect the cyberbully content present in the social network using GenLeven algorithm and to classify the detected cyberbully content using fuzzy rule base [7]. Saravanaraj, et al., had proposed a model for detecting cyberbullying and rumour using naïve bayes and random forest classifier [8]. Imran awan made a study about the cyberbullying based on terrorism. He used a method quantitative data gathering techniques embedded with grounded theory for detecting the cyberbully content. Then vivo tool is used to find the high frequency words which represent the terror and the visualization was done using ncapture tool [9]. Vivek K. Singh, et al., proposed an approach by using the automated image and text analysis APIs for multimodal(visual and textual) cyberbullying detection. The textual detection LIWC (Linguistic Inquiry \&word count) method was used. For visual cyberbullying the Microsoft oxford tool which are developed from the basis of computer vision algorithms was used in it[10]. Soyeon Kim, et al., proposed a model in which they extract the features for cyberbullying, such as power imbalance, repetitiveness, intention to harm by using statistical analysis are taken. Then they categorize the cyberbullying contents into mainly four kinds such as physical, verbal, social bullying and cyberbullying [11]. Calvete,et al., proposed a mechanism of maladaptive schemas for detecting bullying present among adolescent school students. They had made a survey considering factors like age, social anxiety, and family abuse in order to find the continued bullying victimization among adolescents [12]. Santiago Yubero, et al., proposed an approach to analyse the associations between the cyberbullying victimization and social and emotional factors. Such as involvement in traditional bullying victimization. These findings identify the traditional risk factors for bullying in cyberbullying, and also highlight the importance of factors related to peer relationships [13]. Niall McCrae, et al., made a study to find the cyberbullying present among adolescent and analysed the statistically significant correlation between social media use and depressive symptoms in young people [14]. Tata Prathyusha et al., proposed a framework for detecting cyberbullying in the crime investigation forum using hybrid techniques (MCC(Multiple Correlation Coefficient) \& SVM(Support Vector Machine))[15]. Most of the existing system deals only with the detection of textual cyber bullying. The proper implementation techniques are not available for prevention and recovery from the cyber bullying in the form of image, video and audio. To overcome the above problems, in the proposed system is going to detect the multimodel cyber bullying from various techniques in the social network.

\section{PROPOSED FRAMEWORK}

Figure 1 represents a framework for the proposed system which can be explained as follows: The input data set contains text, image, audio and video will be collected from social networks. The input of the data is sent to data preprocessing which improves quality of the input. After completion of the data preprocessing the outcome of the data is sent to cyberbully detection module for detecting the cyberbully contents. Finally the cyberbully content is classified using the classifiers.

In order to detect and prevent the multimodal cyberbullying the following steps are carried out in the proposed framework

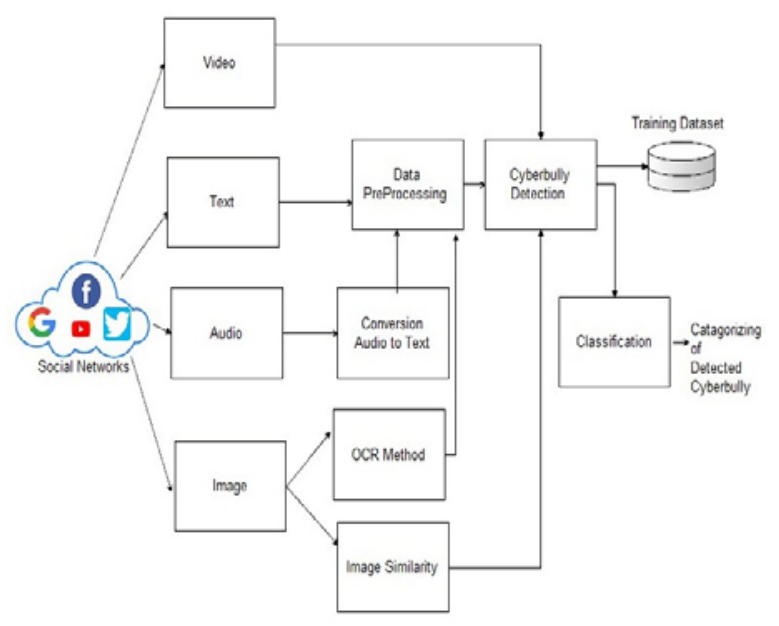

Figure 1: Architecture Diagram for Multimodel Cyberbully Detection

\section{A. Data Preprocessing}

Social network dataset consist of most noisy and unwanted data, to improve the accuracy of the input data the preprocessing has been applied. This includes removing stop words and symbols. Stop words are usually like “a”, "as”, "have”, “is”, “the”, “or”, etc. Stop 
words mainly consume memory space and reduce the processing time.

\section{B. Cyberbully Detection}

The outputofthepreprocessing module will be given input to this module where the cyberbully content such as text, image, audio and video will be detected. The cyberbully detection techniques are explainedbelow:

\section{B.1. Image Cyberbully detection}

Nowadays the cyberbullying using images is vast and causes large effects to the society. They seems to be spreading in the social networks very rapidly. Such as even the anti-social elements able to create more stress to the world by spreading communalism through images. The cyberbully image will be detected using the computer vision algorithm which includes two methods like image similarity and Optical Character Recognition (OCR)[16].

\section{B.2.Video Cyberbully detection}

The video cyberbullying also causes more problems in terms of both emotional and psychological means. The cyberbully video will be detected using the shot boundary detection algorithm. Here the video will be broken into scene, shot and frames. A shot is asequence of frames captured by a single camera in a single continuous action. Thereby using the Shot boundary detection algorithm the content of the video will be analysed using the methods such as Pixel based shot boundary detection, Histogram based shot boundary detection, Block based shot boundary detection [18].

\section{B.3. Audio Cyberbully detection}

The audio is the one of area where many cyberbullying occurs in a larger part. Here the audio will be converted into text using CMU Sphinx tool.In the converted text cyberbully will be detected using trained dataset [17].

\section{C.Classification}

In this module the cyberbully data will be classified into Physical bullying, Social bullying and Verbal bullying using Naïve Bayesian classifier.

\section{Naïve Bayes}

The Naive Bayes classifier method is developed based on Bayesian theorem with assumptions which are independentinbetween predictors [8]. The classifier is tranquil to create, with no complicated iterative parameter estimation which makes ituseful particularly for complex datasets. The classifiers can be highly simplified by considering that features as independent class, that is

$\mathrm{P}(\mathrm{c} \mid \mathrm{x})=\mathrm{P}(\mathrm{x} \mid \mathrm{c}) * \mathrm{P}(\mathrm{c}) / \mathrm{P}(\mathrm{x})$

Where, variable $\mathrm{P}$ is a probability, variable c is a class and variable $\mathrm{x}=\{\mathrm{x} 1, \mathrm{x} 2 \ldots \mathrm{xn}\}$ is a vector[8]. By usingthis classifier the cyberbully contents will be classified into physical, verbal, social bullying.

\section{C.1. Social bullying}

Social bullying involves spreading rumours about a person, purposely embarrassing a person in public where it intends to hurt his or her feeling. The new ideology in the method of bullying that decreases into this grouping involves boosting others to circumvent a definite person or group.Social bullying affects a person and their ability to relate to their environment as well as other people in a social setting. This Technique not only does it have a straight influence on a person's mental and emotional state-owned, it can also undesirably distress their standing in both individual and specialized circles [11].

\section{C.2. Verbal bullying}

Verbal bullying is one of the most highly used techniques to perform bullying mechanism in an efficient way. Criticizing and making fun of othersare all forms of verbal bullying. In verbal bullying the main deterrent the bully uses is their vocal sound. Verbalbullying is defined as a negative aspects based defining declaration told to the victim or about the target, thereby defining the target to be as non-existent one. If the abuser proximatelydoes not make an apology and draw back the significant

declaration, the relationship is considered as verbally abusive one to the network.This will create psychological disorders that plague them into and throughout adulthood periods of an individual[11].

\section{C.3. Physical bullying}

Physical bullying is one in which ones feeling is being hatred or harms their personal possessions. The various types of physical bullying methods which are present widely are Stealing, heaving, hitting, pushing, slapping, spattering and abolishing property [11]. Physical bullying is hardly the primary form of bullying that aBuller will experience. Frequently bullying will commence in analteredmethod and advancement to physical violence. In physical bullying the foremost weapon the bully uses is their body.

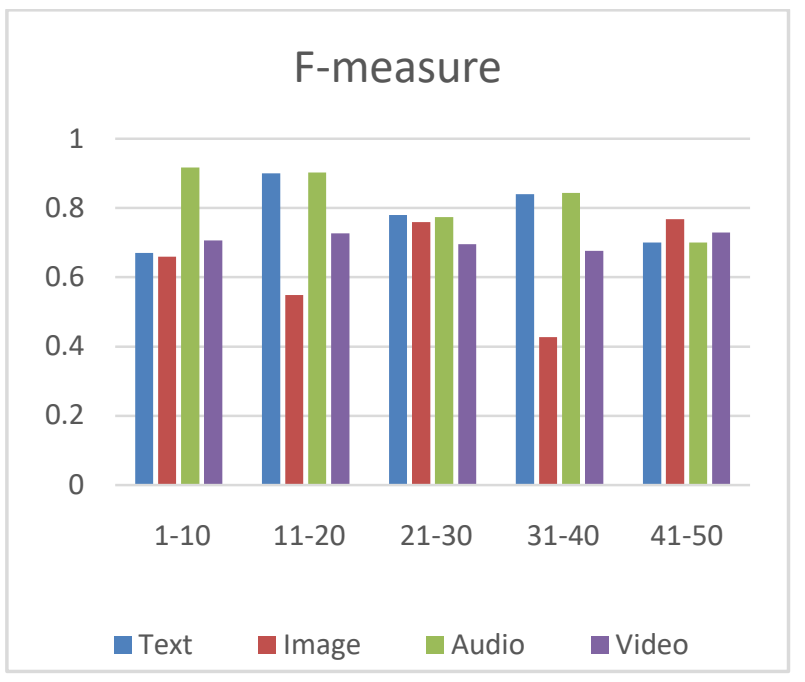


Figure 2: F-measure of cyberbully Detection

Figure 2 shows the F-measure obtained with different inputs text, image, audio, video of cyberbully content detection and classification

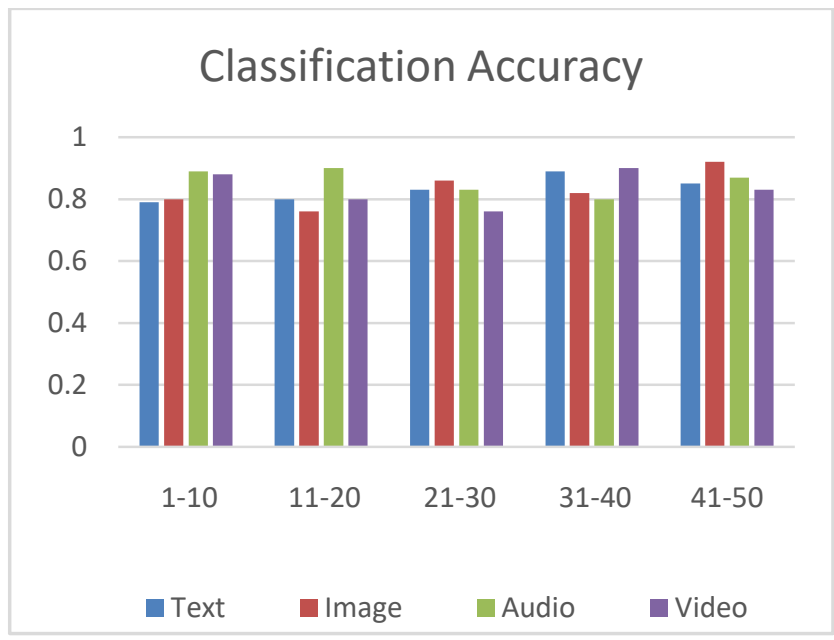

Figure 3: Classification Accuracy of cyberbully Detection

Figure 3 shows the classification accuracy graph obtained using different inputs.

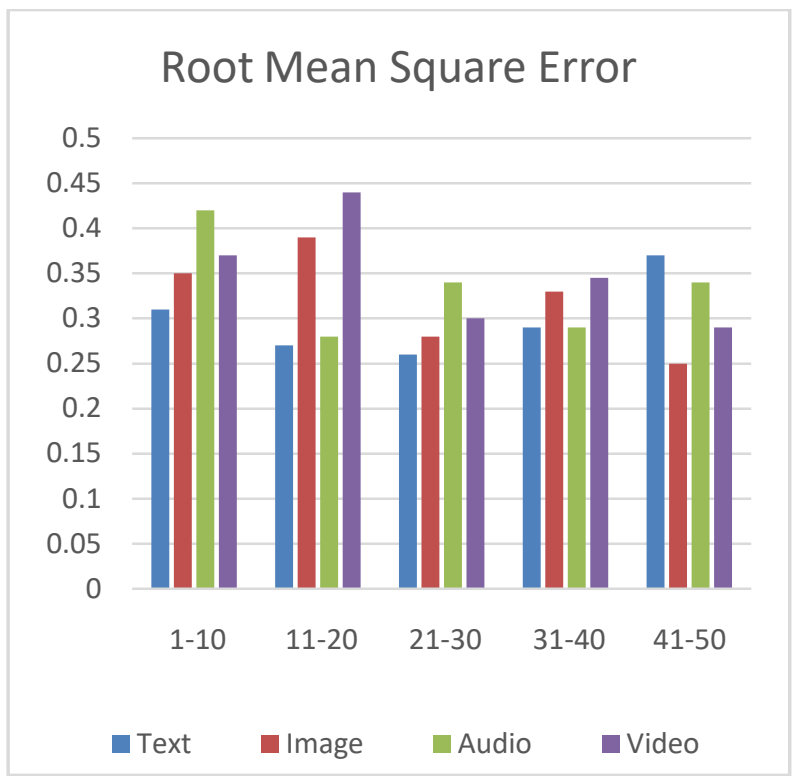

Figure 4: Root mean Square Error of Text cyberbully Detection

Figure 4 shows the RMSE graph in which system using different inputs.

\section{SENSITIVITY}

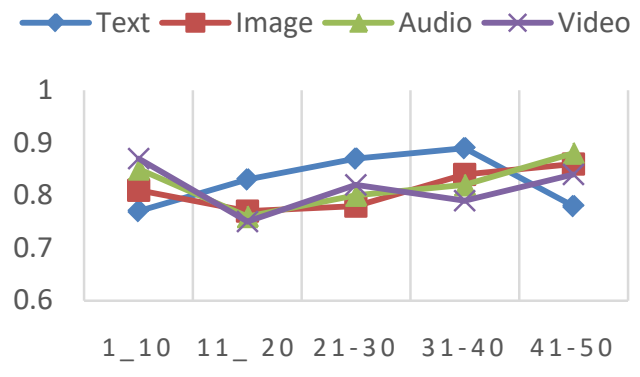

Figure 5: Sensitivity of Cyberbully detection

\section{SPECIFICITY}

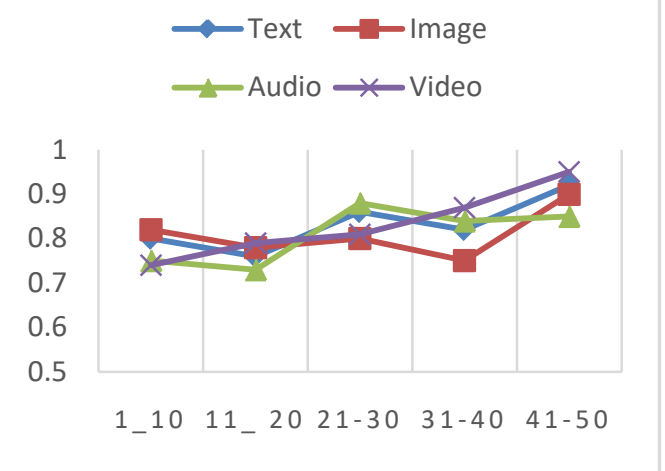

Figure 6: Specificity of cyberbully detection

Sensitivity and specificity values are calculated for different inputs, and the results obtained using the given dataset are shown in Figures 5 and 6.

\section{CONCLUSION}

In an era of internet and social networking, a hectic task of cyberbully detection is made easy by this framework. The proposed system works on detection of cyberbully in image, video and audio from social networks. The detection of cyberbully words will be classified into three categories like Physical bullying, Social bullying and Verbal bullying using naïve bayesclassifier.This proposed framework will showsbetter results while the action is to stop the online users becoming the victims of cyberbully.

\section{REFERENCES}

[1] SheebaJ.I and A.Habiba, "Sentence Abusive Detection using Text Mining." CiiTInternational Journal of Data Mining and Knowledge Engineering, Vol 5.No.7, ISSN(Online):09749578,ISSN(Print):0974-9683,2013 pp: 288-291,2013.

[2] Sheeba, J. I., and K. Vivekanandan. "Detection of Online Social Cruelty Attack from Forums." International Journal of Data Mining And Emerging Technologies 4, no. 2 (2014): 61-71.

[3] Nandhini, B., and J. I. Sheeba. "Cyberbullying detection and classification using information retrieval algorithm." In Proceedings of the 2015 International Conference on 
Advanced Research in Computer Science Engineering \& Technology (ICARCSET 2015), p. 20. ACM, 2015

[4] Nandhini, B. Sri, and J. I. Sheeba. "Online social network bullying detection using intelligence techniques." Procedia Computer Science 45 (2015): 485492.

[5] Kansara, Krishna B., and Narendra M. Shekokar. "A framework for cyberbullying detection in social network." International Journal of Current Engineering and Technology 5 (2015).

[6] Al-garadi, Mohammed Ali, KasturiDewiVarathan, and Sri Devi Ravana. "Cybercrime detection in online communications: The experimental case of cyberbullying detection in the Twitter network." Computers in Human Behavior 63 (2016): 433-443.

[7] J.I.Sheeba,Dr.S.PradeepDevaneyan,“Cyberbully Detection Using Intelligent Techniques”,International Journal of Data Mining and Emerging Technologies, Vol 2.No.2,ISSN(Online):2249-3320, ISSN (Print):22493212,pp.86-94, November 2016.

[8] Saravanaraj, A., J. I. Sheeba, and S. Pradeep Devaneyan. "Automatic Detection of Cyberbullying from Twitter." International Journal of Computer Science and Information Technology \& Security (IJCSITS), ISSN: 2249-9555, Vol.6, No.6, (2016), pp.27-32.

[9] Awan, Imran. "Cyber-extremism: Isis and the power of social media." Society 54, no. 2 (2017): pp.138-149.

[10] Singh, Vivek K., Souvick Ghosh, and Christin Jose. "Toward Multimodal Cyberbullying Detection." In Proceedings of the 2017 CHI Conference Extended Abstracts on Human Factors in Computing Systems, pp. 2090-2099. ACM, 2017.

[11] Kim, Soyeon, Scott R. Colwell, Anna Kata, Michael H. Boyle, and KatholikiGeorgiades. "Cyberbullying victimization and adolescent mental health: evidence of differential effects by sex and mental health problem type." Journal of youth and adolescence (2017): 1-12.
[12] Calvete, Esther, LiriaFernández-González, Joaquín M. González-Cabrera, and Manuel Gámez-Guadix. "Continued bullying victimization in adolescents: maladaptive schemas as a mediational mechanism." Journal of youth and adolescence (2017): 1-11.

[13] Yubero, Santiago, Raúl Navarro, María Elche, Elisa Larrañaga, and AnastasioOvejero. "Cyberbullying victimization in higher education: An exploratory analysis of its association with social and emotional factors among Spanish students." Computers in Human Behavior (2017).

[14] McCrae, Niall, Sheryl Gettings, and Edward Purssell. "Social Media and Depressive Symptoms in Childhood and Adolescence: A Systematic Review." Adolescent Research Review (2017): 1-16.

[15] J.I.Sheeba,TataPrathyusha and R.Hemavathy, "Cyberbully Detection and Classification System Using Hybrid Techniques", In Proceedings of the IEEE International Conference on International Conference On Telecommunication, Power Analysis And Computing Techniques (ICTPACT 2017),April 6-8,Tamil Nadu, India.

[16] Mantoro, Teddy, Abdul MuisSobri, and Wendi Usino. "Optical Character Recognition (OCR) Performance in Server-Based Mobile Environment." In Advanced Computer Science Applications and Technologies (ACSAT), 2013 International Conference on, pp. 423428. IEEE, 2013.

[17] Bravo, Carlos J. Corrada, Rafael ÁlvarezBerríos, and T. Mitchell Aide. "Species-specific audio detection: a comparison of three template-based detection algorithms using random forests." PeerJ Computer Science 3 (2017): e113.

[18] Deokar, Mohini, and RuhiKabra. "Video shot detection techniques brief overview." International Journal of Engineering Research and General Science 2, no. 6 (2014): 817-820. 